\title{
The prognostic significance of PD-L1 in bladder cancer
}

\author{
YIDE HUANG $^{1 *}$, SHU-DONG ZHANG ${ }^{2 *}$, CIAN MCCRUDDEN $^{2}$, KWOK-WAH CHAN ${ }^{3}$, \\ YAO LIN ${ }^{1,4}$ and HANG-FAI KWOK ${ }^{2,4}$ \\ ${ }^{1}$ College of Life Sciences, Fujian Normal University, Fuzhou, Fujian, P.R. China; ${ }^{2}$ Center for Cancer Research \\ and Cell Biology and School of Pharmacy, Queen's University Belfast, Belfast, Northern Ireland, UK; \\ ${ }^{3}$ Department of Pathology, University of Hong Kong, Hong Kong SAR; ${ }^{4}$ Faculty of Health Sciences, \\ University of Macau, Taipa, Macau SAR, P.R. China
}

Received January 8, 2015; Accepted March 23, 2015

DOI: $10.3892 /$ or.2015.3933

\begin{abstract}
Immunotherapy is a promising strategy for the treatment of various types of cancer. An antibody that targets programmed death ligand-1 (PD-L1) pathway has been shown to be active towards various types of cancer, including melanoma and lung cancer. MPDL3280A, an anti-PD-L1 antibody, has shown clear clinical activity in PD-L1-overexpressing bladder cancer with an objective response rate of $40-50 \%$, resulting in a breakthrough therapy designation granted by FDA. These events pronounce the importance of targeting the PD-L1 pathway in the treatment of bladder cancer. In the present study, we investigated the prognostic significance of the expression of three genes in the PD-L1 pathway, including PD-L1, B7.1 and $\mathrm{PD}-1$, in three independent bladder cancer datasets in the Gene Expression Omnibus database. PD-L1, B7.1 and PD-1 were significantly associated with clinicopathological parameters indicative of a more aggressive phenotype of bladder cancer, such as a more advanced stage and a higher tumor grade. In addition, a high level expression of PD-L1 was associated with reduced patient survival. Of note, the combination of PD-L1 and B7.1 expression, but not other combinations of the three genes, were also able to predict patient survival. Our findings support the development of anti-PD-L1, which blocks PD-L1-PD-1 and B7.1-PD-L1 interactions, in treatment of bladder cancer. The observations were consistent in the three independent bladder cancer datasets consisting of a total of 695 human bladder specimens. The datasets were then assessed and it was found that the expression levels of the chemokine $\mathrm{CC}$-motif ligand (CCL), CCL3, CCL8 and CCL18, were correlated with the PD-L1 expression level, while ADAMTS13 was differentially
\end{abstract}

Correspondence to: Professor Hang-Fai Kwok or Professor Yao Lin, Faculty of Health Sciences, University of Macau, Avenida de Universidade, Taipa, Macau SAR, P.R. China

E-mail: hfkwok@umac.mo

E-mail: yaolin@fjnu.edu.cn

${ }^{*}$ Contributed equally

Key words: programmed death ligand-1, bladder cancer, patient survival, prognostic marker, therapeutic targets expressed in patients with a different survival status (alive or deceased). Additional investigations are required to elucidate the role of these genes in the PD-L1-mediated immune system suppression and bladder cancer progression. In conclusion, findings of this study suggested that PD-L1 is an important prognostic marker and a therapeutic target for bladder cancer.

\section{Introduction}

Immunotherapy has been found to be effective for bladder cancer, especially in non-muscle invasive diseases. Treatment of non-muscle invasive disease bladder cancers resulted in a reduction in the risk of recurrence and progression $(1,2)$. Although the mechanisms for BCG in delaying recurrence and prolonging survival remain poorly understood $(2,3)$, the modulation of the expression of a variety of cytokines and immune cell maturation have been demonstrated with BCG treatment even though their functional status and effects remain controversial (1).

Programmed death ligand-1 (PD-L1) is a T-cell regulatory molecule that may be expressed on the surface of tumor and tumor-infiltrating immune cells. The PD-L1/PD-1 pathway has been shown to be important in cancer progression (4). PD-L1 is frequently found to be overexpressed in tumors. By binding to PD-1, it may inhibit the activation of cytotoxic T lymphocytes in order to evade the host immune response preventing tumors from cytotoxic T lymphocyte-induced killing. Other than PD-1, PD-L1 also interacts with B7.1 to further suppress the tumor antigen-induced activation of cytotoxic $\mathrm{T}$ lymphocytes.

Tumor PD-L1 protein expression was found to be associated with a higher grade of tumors and a poorer survival rate in urothelial cancers (5). PD-L1 has been previously identified as an independent determinant for stage progression (6). Among other T-cell co-regulatory molecules, such as B7-H3 and PD-1, investigated in urothelial cell carcinoma of the bladder, only PD-L1 expression levels were found to be associated with an increased pathological stage and an independent predictor for all-cause mortality after cystectomy (7). Microarray technology was recently used to examine the expression of PD-L1, PD-1 and B7-H3 in urothelial carcinoma of the bladder (8). The results of that study showed that the expression levels of the three molecules were correlated with each other in the 
primary tumors, while the expression of PD-L1 was associated with an increased risk of overall mortality in the subgroup of patients with organ-confined disease.

Efforts have begun to delineate the role of the PD-L1 pathway in metastatic urothelial bladder cancer. Inhibition of the PD-L1 pathway by the investigational molecule, MPDL3280A, which blocks PD-1/PD-L1 and B7.1/PD-L1 interactions, thereby completely inhibiting the PD-L1mediated immunosuppressive signals, has shown great clinical activity (9). Patients whose urothelial bladder tumors or their associated tumor-infiltrating lymphocytes had PD-L1 expression of an immunohistochemical (IHC) score of $2+$ or $3+$ had an objective response rate of 40 and $50 \%$, respectively (9). Those results led to a breakthrough therapy designation status for MPDL3280A in the treatment of metastatic urothelial bladder cancer granted by FDA in the USA, indicating that PD-L1 is an important target that should be investigated.

Although IHC is a standard test for protein overexpression, the resulting interpretation is often solely visual and highly subjective. For instance, diagnosis of the HER 2 overexpression levels in breast cancer often requires complementary fluorescence in situ hybridization analysis for those patients with an IHC staining score of $2+$, due to the difficulty of defining a true-positive result, when the staining itself is moderate. Assessment of gene expression at the transcriptional level by quantitative tests removes the subjectivity of interpreting IHC results. In the present study, we aimed to analyze the mRNA expression of related molecules of the PD-L1 pathway and the prognostic significance of the players in the PD-L1 pathway in early bladder cancer, by investigating the associations of the expression of PD-L1, B7.1 and PD-1 and the clinicopathological parameters in three bladder cancer patient cohorts using available microarray and patient data.

\section{Materials and methods}

Extraction of clinical and microarray gene expression data from bladder cancer patient datasets. Three bladder cancer patient datasets, GSE13507 ( $\mathrm{n}=256)(10)$, GSE32894 $(n=308)(11)$ and GSE32548 $(n=131)(12)$ were identified from the Gene Expression Omnibus database based on the following search criteria: i) bladder or urothelial cancer, ii) available information on clinicopathological parameters, iii) available information on the PD-L1, B7.1 and PD-1 gene expression levels and iv) with a sample size of $\geq 100$. The GEO website has standardized URLs for its individual datasets, such as the overall summary information regarding the microarray dataset GSE13507 accessed at http://www.ncbi.nlm.gov/geo/ query/acc.cgi?acc=GSE13507. For each GEO data series, links are provided at the bottom of the page to the Series Matrix File(s), which contain the expression values for each gene (probe set) and each microarray. The URLs to the Series Matrix File(s) are also standardized. For GSE13507, the URL was ftp://ftp.ncbi.nlm.nih.gov/pub/geo/DATA/SeriesMatrix/ GSE13507. The files in gzip format were then unzipped to the tab-delimited text format, which contained detailed information for statistical analysis. R scripting was used to extract the expression values of a small number of genes (probe sets) of interest and the clinical data from the data matrices downloaded from GEO.
Correlations of gene expression levels and clinical data. The statistical analyses were performed using SPSS19.0. The associations between the expression levels of the genes were analyzed by the Spearman's rank test. The expression levels were then divided into high and low levels using the higher quartile expression level as the cut-off point for the Kaplan-Meier survival analysis. The results were compared by the log-rank test. The patients were divided into three groups based on the expression levels of PD-L1 and B7.1. The PD-L1/B7.1 low group comprised patients expressing the two genes at low levels, the PD-L1/B7.1 intermediate group comprising patients expressing one of the two genes at high levels, and the PD-L1/B7.1 high group comprising patients expressing the two genes at high levels. The survival time of the patients stratified by this grouping method was analyzed by the Kaplan-Meier survival analysis as described above. The univariate Cox regression and multivariate analyses were performed for the clinicopathological parameters, including gender, age, T-stage, tumor type and grade and PD-L1 expression. The multivariate Cox regression analysis with forward stepwise selection and an entry limit of $\mathrm{p}<0.05$ was performed to identify independent predictors of survival in the patient cohort. Muscle invasive bladder cancer specimens were examined using the Chi-square test.

Identification of PD-L1 co-expressing genes. The patients were stratified into two groups based on the expression levels of PD-L1 as described above. The gene expression patterns of patients in the PD-L1 low subgroup and those in the PD-L1 high subgroup (whose survival was significantly poorer) were compared. Probe sets that were differentially expressed between the two subgroups were identified by the two-sample Welch's t-test. This test was used to avoid the type I error due to unequal variances of the values of the probe sets between the subgroups. Briefly, a Welch's t-test was applied to each probe set corresponding to a certain gene in the data matrix using our own Java application MyStats. P-values and the differential expression in fold-changes for all the probe sets were generated as tab-delimited worksheets of Excel for subsequent analysis.

\section{Identification of therapeutic targets for bladder cancer patients} overexpressing PD-L1. Patients who had tumors expressing a high level of PD-L1 were stratified into two groups based on their survival status (alive or deceased). Differential expression of different probe sets between patients in the PD-L1 high-alive subgroup and those in the PD-L1 high-deceased subgroup were identified as described above.

\section{Results}

Association between the expression levels of PD-L1, B7.1 and $P D-1$ with clinicopathological parameters and survival in bladder cancer. Three independent microarray datasets were identified in the GEO database when we limited our search to include only bladder cancer datasets with a sample size of $>100$ patients and available clinical data, including survival and tumor grade. The three datasets were GSE13507 $(n=256)$, GSE32894 $(n=308)$ and GSE32548 $(n=131)$. The expression levels of PD-L1, B7.1 and PD-1 were extracted and correlated with available clinical data for the three datasets. 
A

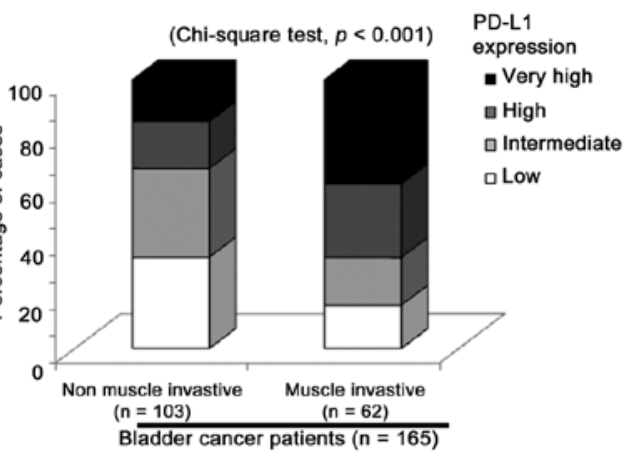

C

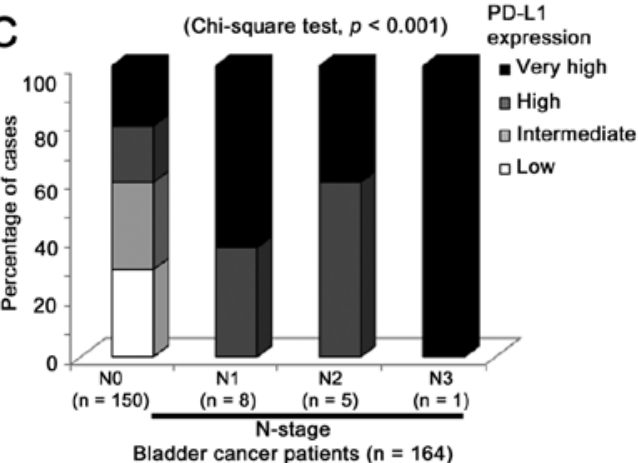

E

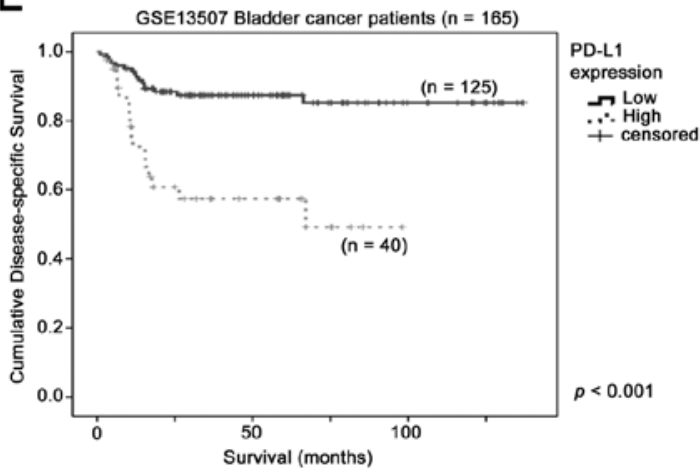

B
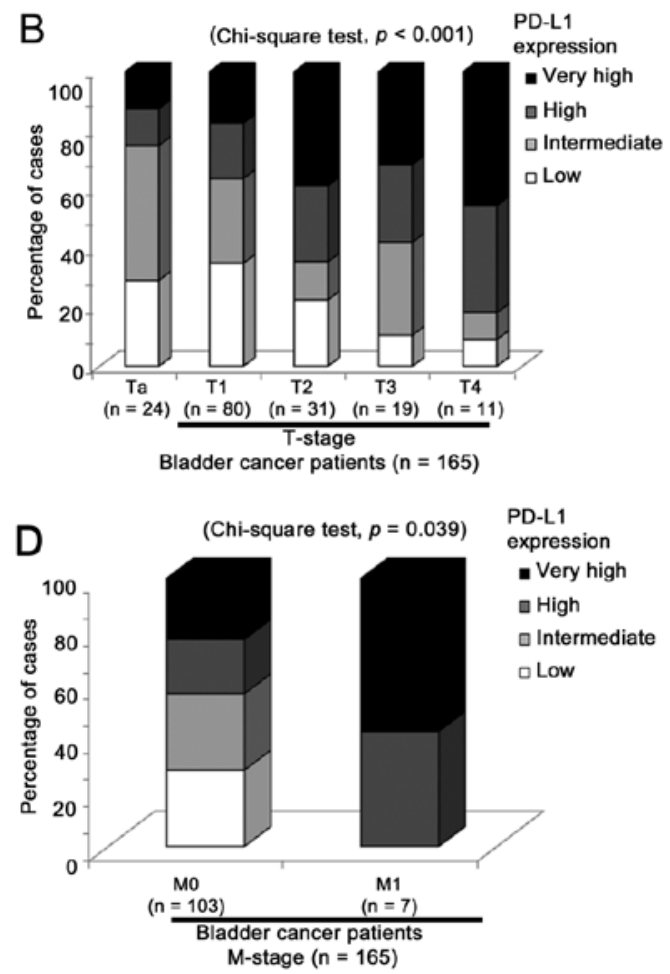

F

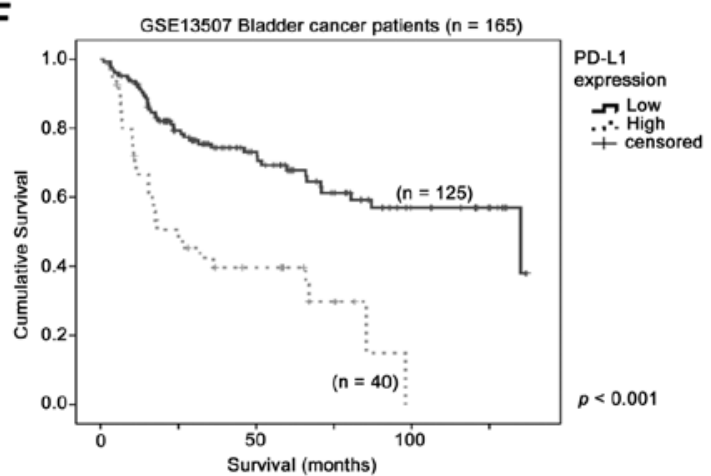

Figure 1. The association between PD-L1 expression and clinicopathological parameters in GSE13507 cohorts with 165 bladder cancer specimens. Histograms showing the percentage of cases with different PD-L1 expression according to (A) the muscle invasive status of the tumors, (B) the T stage of the tumors, (C) the $\mathrm{N}$ stage of the tumors, and (D) the M stage of the tumors. Kaplan-Meier plots for disease-specific survival for patients with (E) differential PD-L1 expression, and (F) for overall survival for patients with differential PD-L1 expression. PD-L1, programmed death ligand-1.

In GSE13507 ( $\mathrm{n}=256)(10)$, PD-L1 and B7.1 expression did not vary significantly between normal bladder $(n=10)$, surrounding mucosa $(n=58)$, bladder cancer $(n=165)$ and recurrent tumor $(n=23)$, while the expression of PD-1 was significantly reduced in bladder cancer compared to normal bladder or surrounding mucosa (Welch's t-test, $\mathrm{p}<0.001$; post-hoc Games-Howell test, $\mathrm{p}=0.024$ and $\mathrm{p}=0.001$, respectively). Muscle invasive bladder cancer specimens expressed significantly more PD-L1 (Fig. 1A; Chi-square test, $\mathrm{p}<0.001$ ) and B7.1 (data not shown). The expression levels of PD-L1 were significantly higher with a higher T stage (Fig. 1B; Chi-square test, $\mathrm{p}<0.001)$ and were also significantly higher with a higher $\mathrm{N}$ stage of the tumors (Fig. 1C; Chi-square test, $\mathrm{p}<0.001$ ). The expression levels of the three genes were significantly higher in tumors of high grade than those of low grade (data not shown). In addition, the mRNA expression of PD-L1 was significantly higher (Welch's t-test, $\mathrm{p}=0.039$ ) in primary tumors with M1 $(n=7)$ stage than those with M0 stage $(n=158)$, although the number of specimens with M1 stage was small (Fig. 1D). We also investigated whether PD-L1 expression was associated with patient survival by stratifying the cohort into two sub-cohorts based on their PD-L1 expression. One sub-cohort comprised the quartile of patients with the highest PD-L1 expression, and the second sub-cohort that of the remaining three quartiles. Notably, the high expression of PD-L1 mRNA (top 25\%) in the primary tumors was significantly associated with a reduced overall survival time (Fig. 1E; log-rank test, $\mathrm{p}<0.001)$ and a shorter disease-specific survival time of patients (log-rank test, $\mathrm{p}<0.001)$. No significant association was observed for the expression of B7.1 or PD-1.

In GSE32894 ( $n=308)(11)$, we found that the expression level of PD-L1 was significantly higher in tumors of higher stage (Fig. 2A, p<0.001) and grade (Fig. 2B, p=0.005). The expression levels of B7.1 and PD-1 were also significantly 

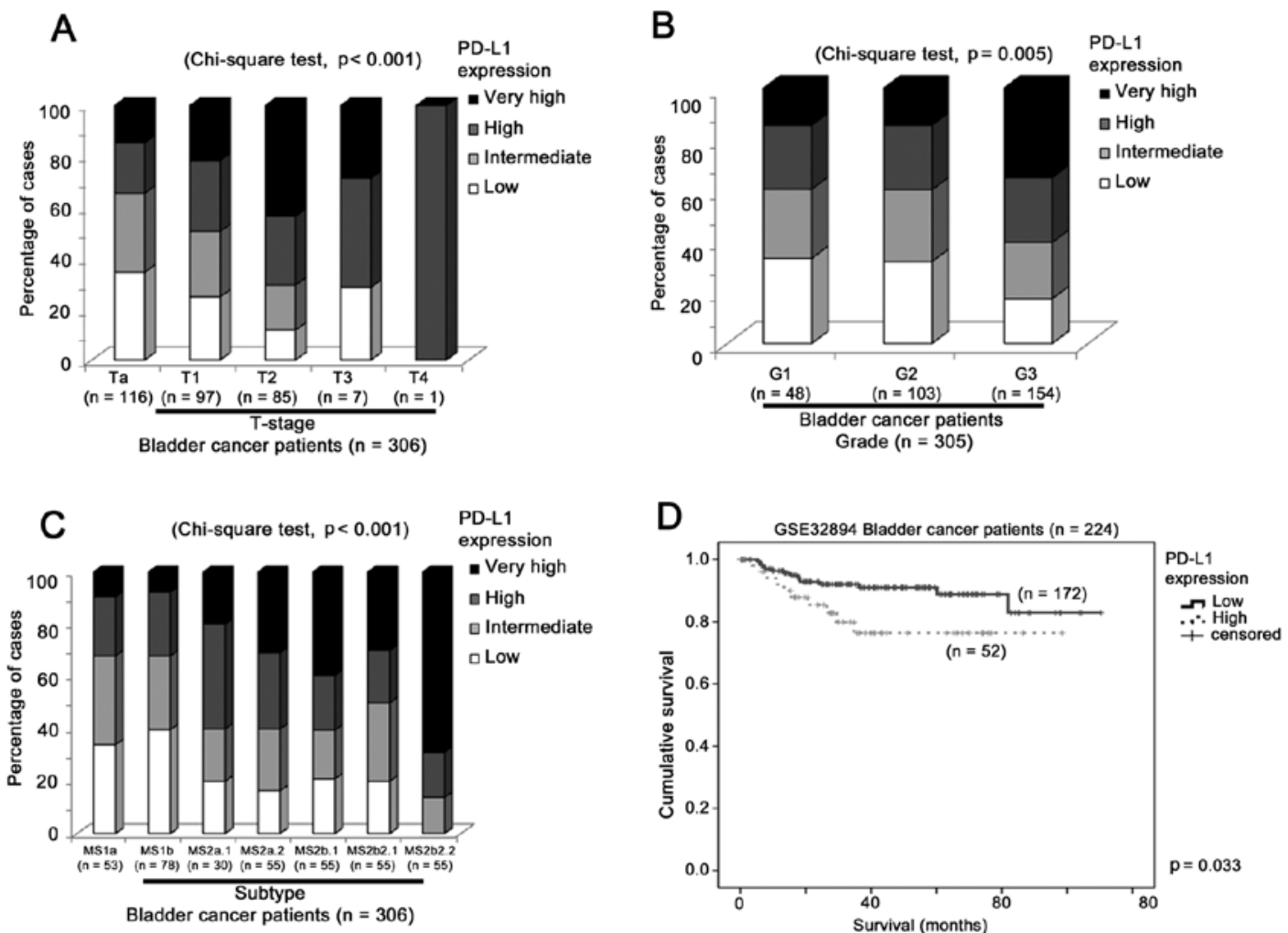

Figure 2. The association between PD-L1 expression and clinicopathological parameters in GSE32894 cohorts with 308 bladder cancer specimens. Histograms showing (A) the percentage of cases with a different PD-L1 expression according to the T-stage of the tumors, (B) the percentage of the cases with different PD-L1 expression according to the histological grading of the tumors, and (C) the percentage of cases with different PD-L1 expression according to the molecular subtype of the tumors. (D) Kaplan-Meier analysis for overall survival for patients with differential PD-L1 expression. PD-L1, programmed death ligand-1.

A

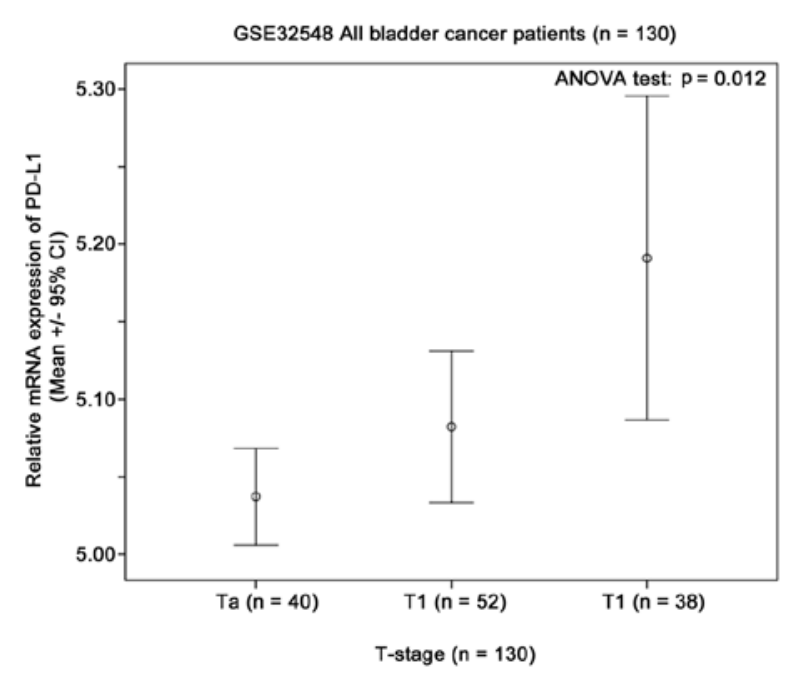

B

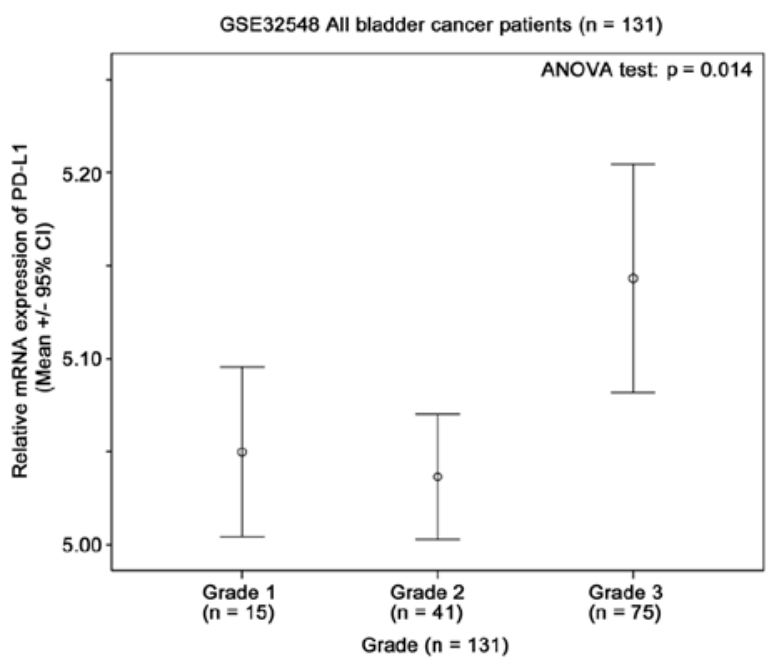

Figure 3. The association between PD-L1 expression and the clinicopathological parameters in GSE32548 cohorts with 131 bladder cancer specimens. Error bar plots for the PD-L1 expression in tumors with a different (A) T stage, and (B) histological grading. PD-L1, programmed death ligand-1.

higher in tumors of a higher stage and grade (data not shown). The mRNA expression of PD-L1 (Fig. 2C, p<0.001) and B7.1 (data not shown), but not that of PD-1 (data not shown), was significantly higher in subtype MS2b2.2, which is one of the two subtypes with a poorer prognosis compared to the remaining five subtypes (11). Similar to the other datasets, a high level of PD-L1 (top 25\%) expression was significantly associated with a shorter overall survival time (Fig. 2D; logrank test, $p=0.033$ ). Again, this association was not observed for B7.1 or PD-1 expression. 
A

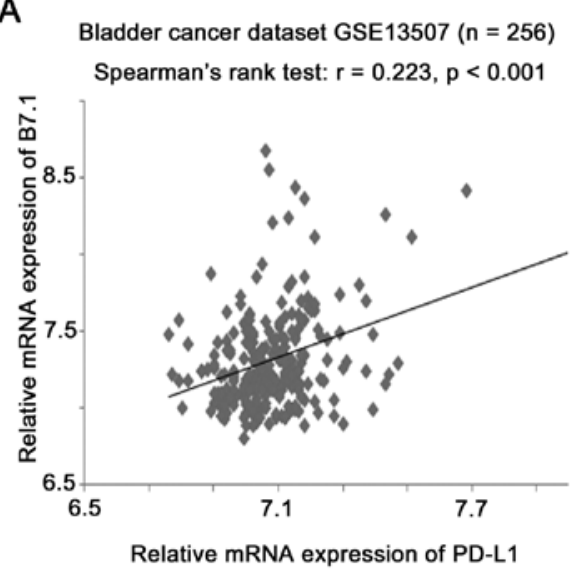

C

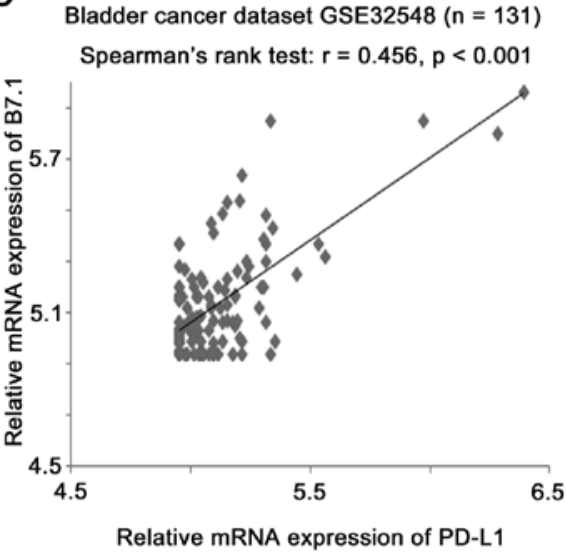

$E$

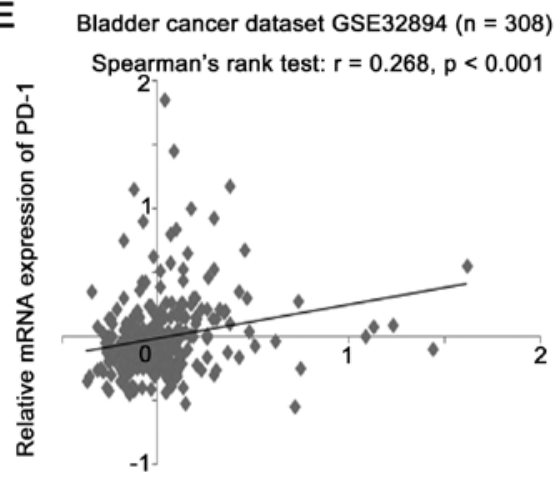

Relative mRNA expression of PD-L1
B

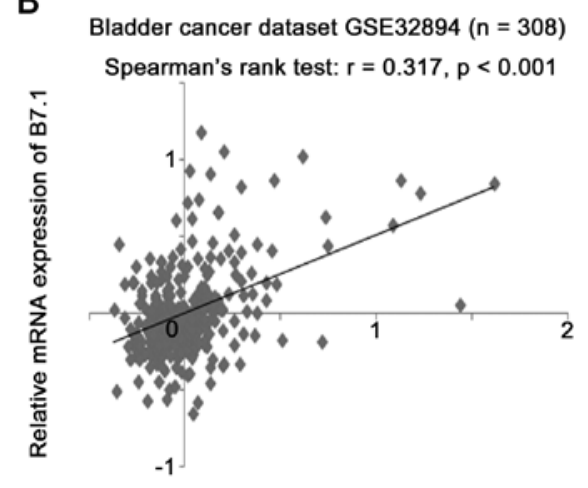

Relative mRNA expression of PD-L1

D

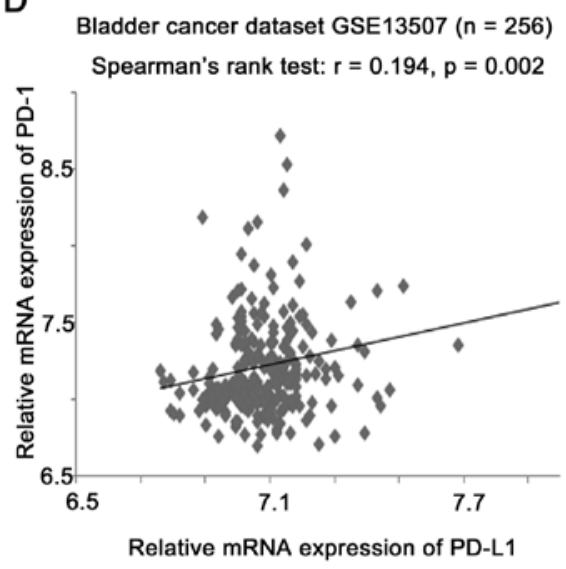

F Bladder cancer dataset GSE32548 $(n=131)$

Spearman's rank test: $r=0.285, p=0.001$

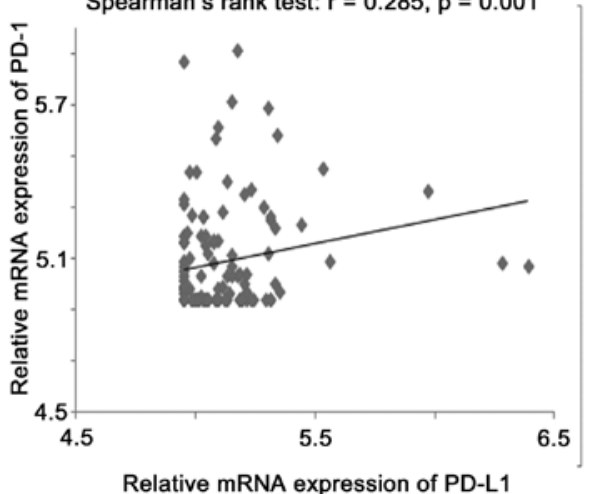

Figure 4. The correlation between PD-L1 and B7.1, and between PD-L1 and PD-1 in the three bladder cancer datasets. Scatter plots showing the expression of PD-L1 vs. B7.1 in (A) GSE13507, (B) GSE32894 and (C) GSE32548, and the expression of PD-L1 vs. PD-1 in (D) GSE13507, (E) GSE32894 and (F) GSE32548. PD-L1, programmed death ligand-1.

In GSE32548 ( $\mathrm{n}=131)(12)$, the PD-L1 mRNA expression level was significantly higher in patients with a higher T-stage (Fig. 3A, p=0.012) and tumor grade (Fig. 3B, p=0.014). The same was true for B7.1 and PD-1, the expression of which was enhanced with the increasing T-stage and tumor grade (data not shown).

The results suggested that a high level of the PD-L1 expression predicts a poor prognosis of bladder cancer patients in terms of patient survival. While an association between the B7.1 expression and the survival of bladder cancer patients was not demonstrated, its high level of expression was associated significantly with tumors with more aggressive phenotypes.
Correlations between the expression of PD-L1, B7.1 and PD-1. The correlations of the mRNA expression of the three genes were then studied to determine whether they are co-overexpressed in bladder cancer. The three genes were highly correlated with each other in the radical cystectomy specimens in the three datasets analyzed (Fig. 4). In GSE13507 ( $n=256$ ), the expression of PD-L1 was significantly positively correlated with that of B7.1 and PD-1 (Fig. 4A and D; Spearman's rank test, $r=0.233, p<0.001$; and $r=0.194, p=0.002$, respectively), while the B7.1 expression also significantly positively correlated with the PD-1 expression (data not shown). In GSE32894 $(n=308)$, the expression of PD-L1 was significantly posi- 

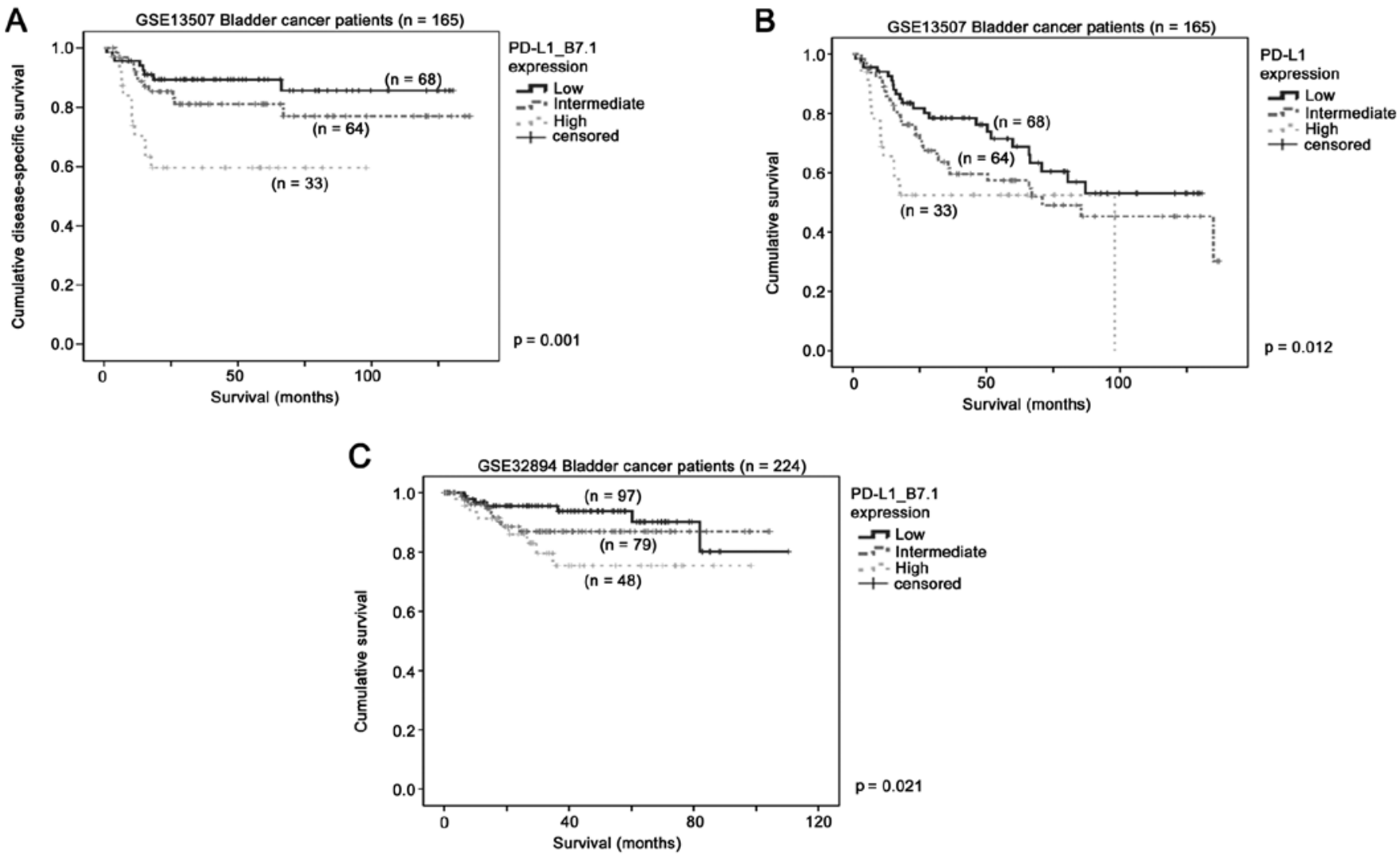

Figure 5. The association between the combination of PD-L1 and B7.1 expression and bladder cancer survival. Kaplan-Meier plots for (A) disease-free survival, (B) overall survival in the GSE13507 cohort, and (C) overall survival in the GSE32894 cohort, of patients with different PD-L1 and B7.1 expression. PD-L1, programmed death ligand-1.

tively correlated with that of B7.1 and PD-1 (Fig. 4B and E; Spearman's rank test, $\mathrm{r}=0.317$, $\mathrm{p}<0.001$; and $\mathrm{r}=0.268, \mathrm{p}<0.001$, respectively), while the $\mathrm{B} 7.1$ expression was also significantly positively correlated with the PD-1 expression (data not shown). In GSE32548 ( $n=131)$, the expression of PD-L1 was significantly positively correlated with that of B7.1 and PD-1 (Fig. 4C and F; Spearman's rank test, $r=0.456, \mathrm{p}<0.001$; and $\mathrm{r}=0.285, \mathrm{p}=0.001$, respectively), while the $\mathrm{B} 7.1$ expression was also significantly positively correlated with the PD-1 expression (data not shown). These results suggested that the mRNA expression of PD-L1 is highly correlated with that of B7.1 and PD-1.

Association of co-overexpression of PD-L1 and B7.1, and survival in bladder cancer. We investigated whether the correlations between PD-L1 and B7.1, and between PD-L1 and PD-1 may be of prognostic significance. Patients expressing high levels of PD-L1 and B7.1 had a shorter survival time than those patients expressing low levels of PD-L1 and B7.1 (Fig. 5). In GSE13507, patients with bladder cancer expressing high levels of PD-L1 and B7.1 had a mean overall survival of 62 months compared to 115 months for those with bladder cancer expressing low levels of PD-L1 and B7.1 (Fig. 5A; logrank test, $\mathrm{p}=0.001)$. Similarly, patients with bladder cancer expressing high levels of the two genes had a mean disease-free survival of only 56 months, which was significantly shorter than 89 months for those patients with bladder cancer expressing low levels of the two genes (Fig. 5B; log-rank test, $p=0.012$ ). In addition, similar results were obtained in GSE32894. Patients with bladder cancer expressing high level of the two genes had a mean survival of 79 months, which was significantly shorter than 100 months for those patients with their bladder cancers expressing low levels of the two genes (Fig. 5C; log-rank test, $\mathrm{p}=0.021)$. However, no significant association was detected for the co-expression of PD-L1 and PD-1 or that of B7.1 and PD-1. These results suggested that the co-overexpression of PD-L1 and B7.1, but not that of the other combinations, is important for bladder cancer progression.

PD-L1 expression is an independent predictor for survival in bladder cancer. Since the PD-L1 expression was the most significant factor among PD-L1, PD-1 and B7.1, in predicting survival in bladder cancer, we investigated whether the association between PD-L1 expression and survival is independent of other predictive clinicopathological parameters. Since the GSE13507 dataset had a high sample number as well as a high event rate, this dataset was optimal for identifying independent predictive factors. As shown in Tables I and II, using multivariate Cox regression analysis, $\mathrm{PD}-\mathrm{L} 1$ expression, T stage, tumor type and age were independent predictors for disease-specific survival, while for PD-L1 expression, T stage and age were independent predictors for overall survival, respectively. This analysis suggested that the PD-L1 expression is an important prognostic factor in bladder cancer independent of the $\mathrm{T}$ stage as well as whether it is muscle invasive or not.

Identification of PD-L1 co-regulated genes in bladder cancer. We investigated the differential gene expression between 
Table I. Cox regression analysis of disease-specific survival in GSE13507 bladder cancer dataset.

\begin{tabular}{|c|c|c|c|c|}
\hline \multirow[b]{2}{*}{ Clinicopathological variables } & \multicolumn{2}{|c|}{ Univariate analysis } & \multicolumn{2}{|c|}{ Multivariate analysis } \\
\hline & Hazard ratio $(95 \% \mathrm{CI})$ & P-value & Hazard ratio $(95 \% \mathrm{CI})$ & P-value \\
\hline \multicolumn{5}{|l|}{ Gender } \\
\hline Female $(n=30)$ & 1 & Reference & & \\
\hline Male $(n=135)$ & $0.477(0.220-1.032)$ & 0.060 & & \\
\hline Age $($ years $)(n=165)$ & $1.051(1.016-1.088)$ & 0.004 & $1.061(1.020-1.096)$ & 0.001 \\
\hline \multicolumn{5}{|l|}{ T stage } \\
\hline Early $(\mathrm{n}=135)$ & 1 & Reference & 1 & Reference \\
\hline Advanced $(n=30)$ & $12.747(6.129-26.509)$ & $<0.001$ & $3.261(1.464-7.267)$ & 0.004 \\
\hline \multicolumn{5}{|l|}{ Tumor type } \\
\hline Non-muscle invasive $(n=103)$ & 1 & Reference & 1 & Reference \\
\hline Muscle invasive $(\mathrm{n}=62)$ & $24.719(7.501-81.457)$ & $<0.001$ & $13.342(3.556-50.065)$ & $<0.001$ \\
\hline \multicolumn{5}{|l|}{ Grade } \\
\hline Low $(n=105)$ & 1 & Reference & & \\
\hline High $(n=60)$ & $5.980(2.755-12.980)$ & $<0.001$ & & \\
\hline \multicolumn{5}{|l|}{ PD-L1 expression } \\
\hline Low $(n=125)$ & 1 & Reference & 1 & Reference \\
\hline High $(n=40)$ & $4.049(2.020-8.117)$ & $<0.001$ & $2.972(1.442-6.127)$ & 0.003 \\
\hline
\end{tabular}

PD-L1, programmed death ligand-1. Numbers in bold indicate statistical significance $(\mathrm{p}<0.05)$.

Table II. Cox regression analysis of overall survival in GSE13507 bladder cancer dataset.

\begin{tabular}{|c|c|c|c|c|}
\hline \multirow[b]{2}{*}{ Clinicopathological variables } & \multicolumn{2}{|c|}{ Univariate analysis } & \multicolumn{2}{|c|}{ Multivariate analysis } \\
\hline & Hazard ratio $(95 \% \mathrm{CI})$ & P-value & Hazard ratio $(95 \% \mathrm{CI})$ & P-value \\
\hline \multicolumn{5}{|l|}{ Gender } \\
\hline Female $(\mathrm{n}=30)$ & 1 & Reference & & \\
\hline Male $(n=135)$ & $0.641(0.361-1.138)$ & 0.129 & & \\
\hline Age (years) $(n=165)$ & $1.070(1.044-1.096)$ & $<0.001$ & $1.066(1.039-1.093)$ & $<0.001$ \\
\hline \multicolumn{5}{|l|}{ T stage } \\
\hline Early $(\mathrm{n}=135)$ & 1 & Reference & 1 & Reference \\
\hline Advanced $(n=30)$ & $4.400(2.611-7.414)$ & $<0.001$ & $4.576(2.668-7.846)$ & $<0.001$ \\
\hline \multicolumn{5}{|l|}{ Tumor type } \\
\hline Non-muscle invasive $(n=103)$ & 1 & Reference & & \\
\hline Muscle invasive $(\mathrm{n}=62)$ & $2.897(1.791-4.684)$ & $<0.001$ & & \\
\hline \multicolumn{5}{|l|}{ Grade } \\
\hline Low $(n=105)$ & 1 & Reference & & \\
\hline High $(n=60)$ & $2.740(1.694-4.433)$ & $<0.001$ & & \\
\hline \multicolumn{5}{|l|}{ PD-L1 expression } \\
\hline Low $(n=125)$ & 1 & Reference & 1 & Reference \\
\hline High $(n=40)$ & $3.025(1.850-4.946)$ & $<0.001$ & $2.273(1.370-3.771)$ & 0.001 \\
\hline
\end{tabular}

PD-L1, programmed death ligand-1. Numbers in bold indicate statistical significance $(\mathrm{p}<0.05)$.

bladder cancer expressing a high level of PD-L1 and those expressing a low level of PD-L1. Of the genes that were correlated with PD-L1 expression in bladder cancer, three chemokine (C-C motif) ligand (CCL) genes, CCL3, CCL8 and CCL18, were among the top 30 differentially expressed genes with some of the lowest $\mathrm{p}$-values found during the comparison. 

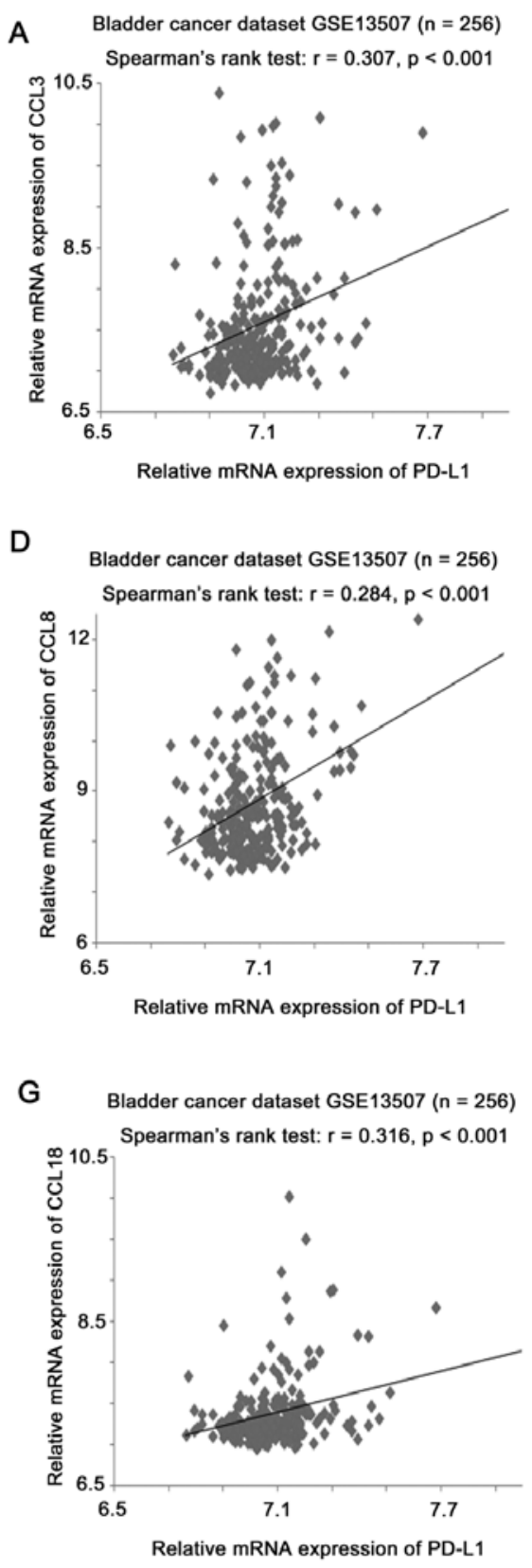
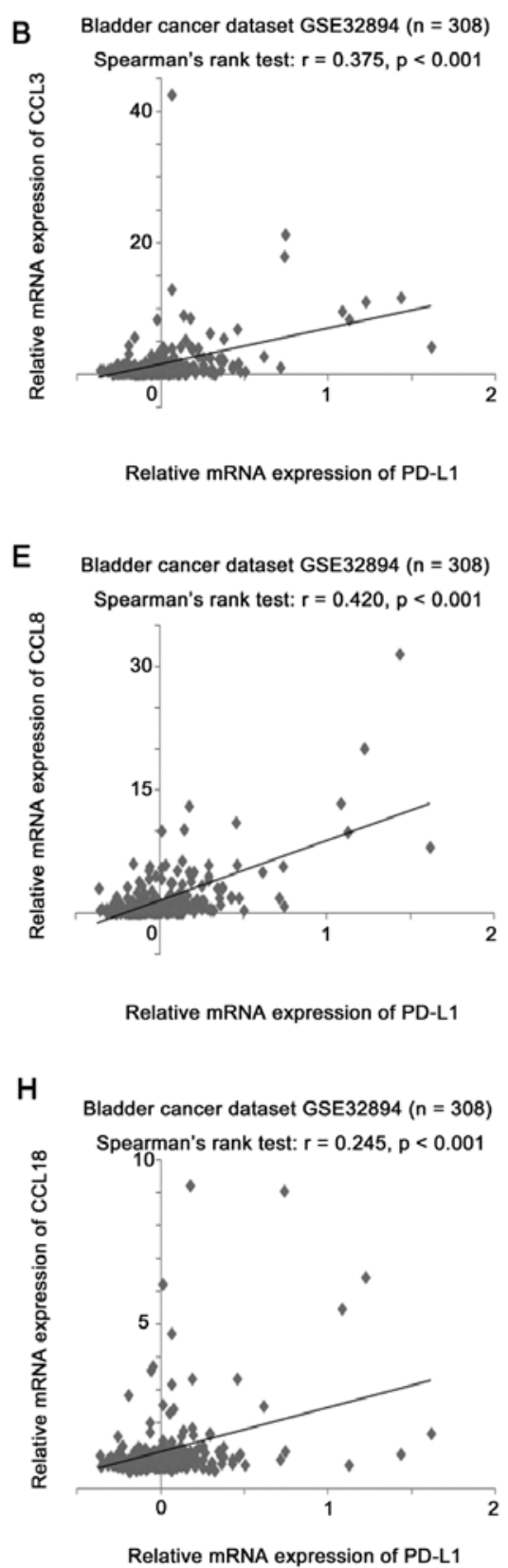
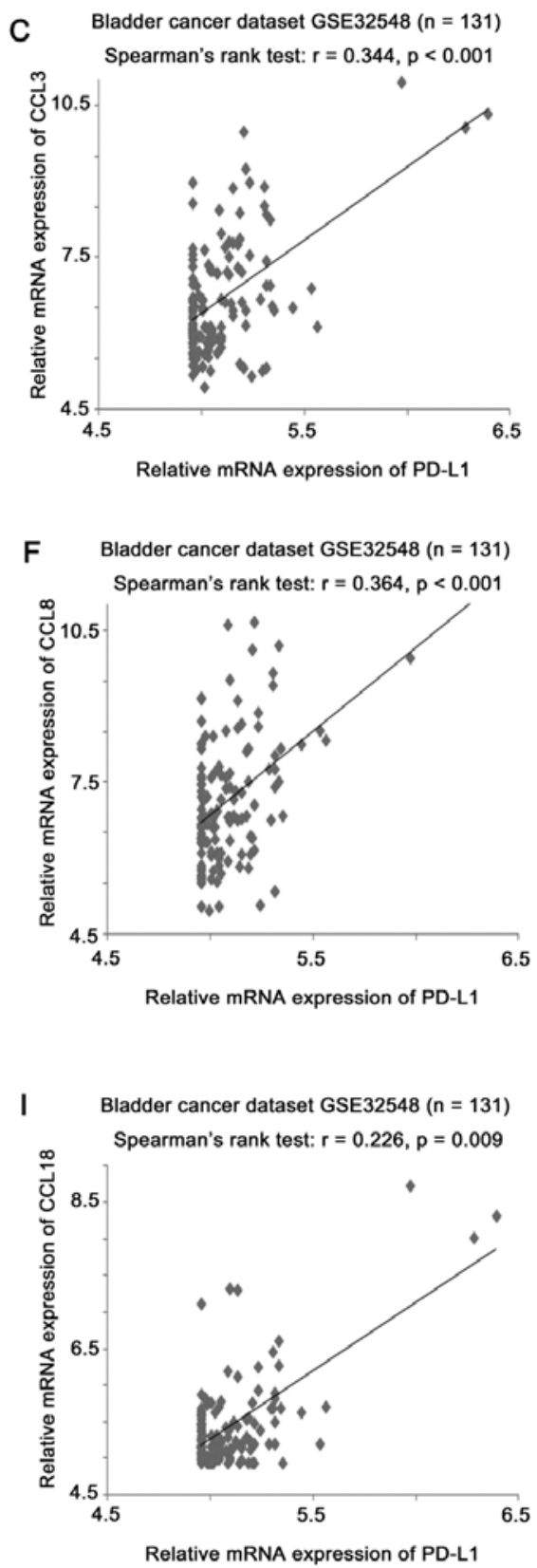

Figure 6. The correlation between PD-L1 and CCLs in the three bladder cancer datasets. Scatter plots showing the expression of PD-L1 vs. CCL3 in (A) GSE13507, (B) GSE32894 and (C) GSE32548, the expression of PD-L1 vs. CCL8 in (D) GSE13507, (E) GSE32894 and (F) GSE32548, and he expression of PD-L1 vs. CCL18 in (G) GSE13507, (H) GSE32894 and (I) GSE32548. PD-L1, programmed death ligand-1; CCL, chemokine CC-motif ligand.

Since CCLs have been suggested to play an important role in malignant growth and metastasis (13), the correlations between PD-L1 expression and the CCL3, CCL8 and CCL18 expression levels were investigated by the Spearman's rank test. In GSE13507, PD-L1 expression was significantly positively correlated with the CCL3 (Fig. 6A; r=0.307, p<0.001), CCL8 (Fig. 6D; r=0.284, p<0.001) and CCL18 (Fig. 6G; $\mathrm{r}=0.316, \mathrm{p}<0.001)$ expression levels. In GSE32894, PD-L1 expression was significantly positively correlated with CCL3 (Fig. 6B; r=0.375, p<0.001), CCL8 (Fig. 6E; r=0.420, p<0.001) and CCL18 (Fig. 6H; r=0.245, p<0.001) expression levels. In GSE32548, PD-L1 expression was significantly positively correlated with CCL3 (Fig. 6C; $r=0.344, p<0.001$ ), CCL8 (Fig. 6F; r=0.364, p<0.001) and CCL18 (Fig. 6I; $\mathrm{r}=0.226, \mathrm{p}=0.009$ ) expression levels.
Identification of genes that predict survival in patients with high level expression of PD-L1. We investigated differentially expressed genes in patients with a high level of PD-L1 between those who were still alive and those who were deceased at the last follow-up. Patient survival data were available in two of the datasets in the present study. Only the ADAMTS13 expression was shown to confer further prognostic value in those patients with bladder cancer already expressing a high level of PD-L1, consistently, in the two datasets with the survival time available. A lower median expression of the ADAMTS13 gene was significantly associated with a shorter overall survival time in the GSE13507 (Fig. 7B; log-rank test, p=0.003) and GSE32894 (Fig. 7D; log-rank test, $\mathrm{p}=0.006$ ) datasets only in patients whose bladder cancer expressed a high level of PD-L1, but not in the other patients (Fig. 7B and D). 
A

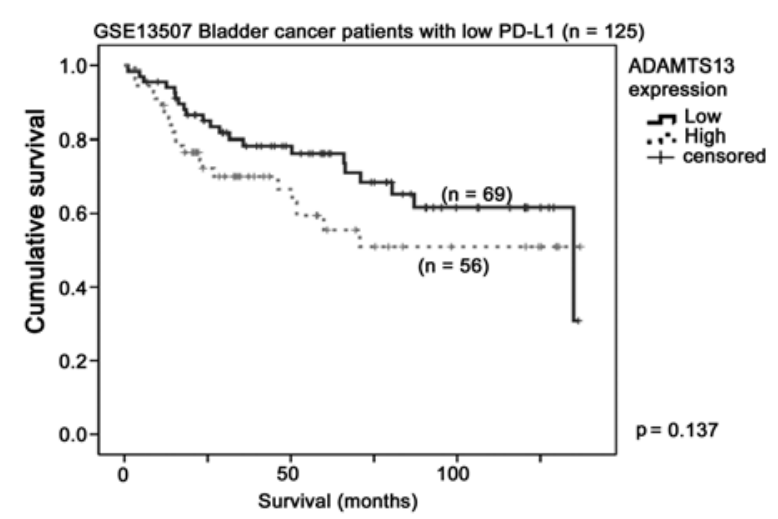

C

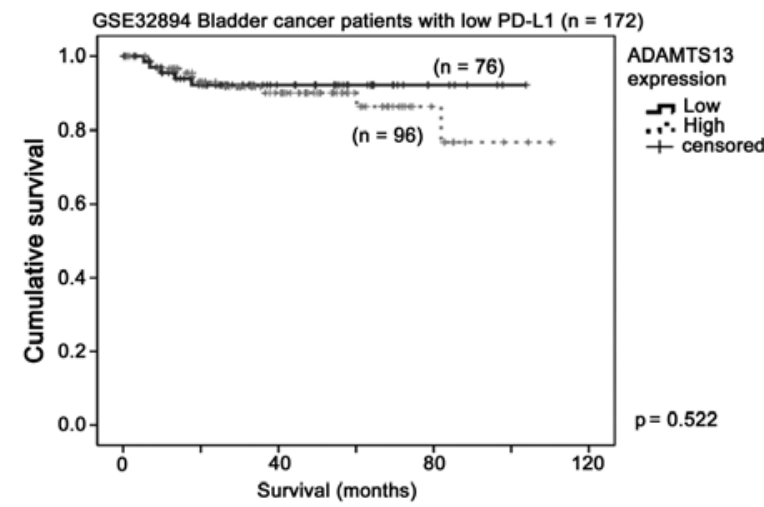

B
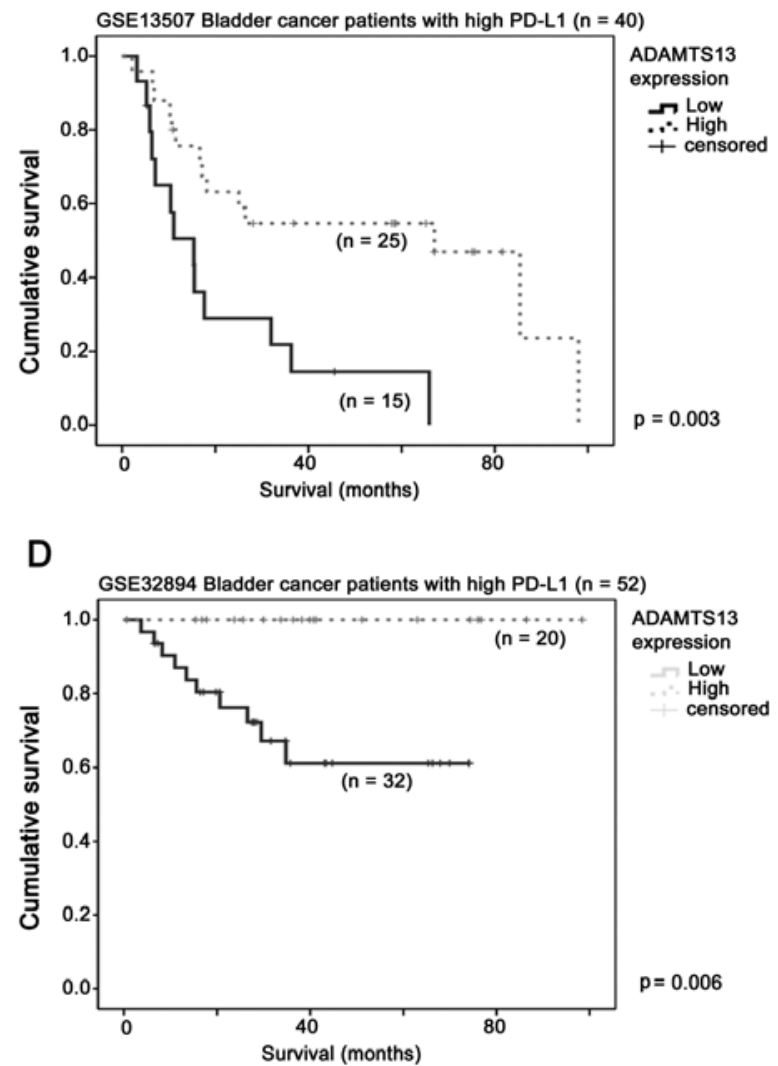

Figure 7. The association between ADAMTS13 expression and bladder cancer survival in patients with different PD-L1 expression Kaplan-Meier plots for overall survival for the differential expression of ADAMTS13 in patients with a (A) low or (B) high level of expression of PD-L1 in the GSE13507 patient cohort, and in patients with a (C) low or (D) high level of expression of PD-L1 in the GSE32894 patient cohort. PD-L1, programmed death ligand-1.

\section{Discussion}

The PD-L1 pathway is important in cancer immunosurveillance (14), and is an important target for cancer immunotherapy (15). MPDL3280A, a monoclonal antibody targeting PD-L1, has shown promising response in the treatment of bladder cancer in a phase I study (9). In the present study, we have shown that the expression of PD-L1, B7.1 and PD-1 was associated with more aggressive clinicopathological parameters of bladder cancer in three independent cancer datasets, a highly consistent observation. Additionally, a high level of expression of PD-L1, but not that of B7.1 and PD-1, was associated with a shorter survival time in two independent bladder cancer datasets, suggesting that PD-L1 is a potential prognostic factor and a promising therapeutic target in bladder cancer.

The expressions of PD-L1, B7.1 and PD-1 were significantly correlated with each other. Thus, we investigated the prognostic significance of their entwining relationships. Only the PD-L1 and B7.1 co-overexpression was significantly associated with a shorter survival time in bladder cancer patients. The latter finding is important, as there is an important difference between the antibody targeting PD-L1 and PD-1, i.e., while the anti-PD-L1 antibody may be able to block the PD-L1-PD-1 and PD-L1-B7.1 interactions, the anti-PD-1 antibody would be ineffective against the PD-L1-B7.1 interaction $(9,16)$, thus rationalizing the use of an anti-PD-L1 antibody, MPDL3280A, in clinical trials for the treatment of bladder cancer.
CCL3 has been shown to be produced by myeloid cells from bladder cancer patients, while expansion of these myeloid cells is associated with cancer-related inflammation and tumor-induced immune suppression (17). The urinary concentration of CCL18 has been shown to be significantly elevated in bladder cancer patients and a highly specific biomarker for the early detection of bladder cancer $(18,19)$. Since CCL18 expression is mainly detected in inflammatory cells in bladder tumor specimens (20), it follows that a high level of expression of CCLs in bladder cancer specimens may reflect the amount of tumor infiltrating lymphocytes. Tumor-infiltrating lymphocytes are an important phenomenon implicating activation of the immune system for the killing of tumor cells, which has been shown to correlate with patient survival (21). However, the overexpression of PD-L1 has been shown to be common in tumor cells and infiltrating lymphocytes, and this suppresses the activation of these immune cells and their ability to kill tumor cells $(4,22)$. In the present study, we have shown that the expression of PD-L1 in bladder cancer specimens was significantly positively correlated with the expression of CCLs, and we hypothesize that PD-L1 expression in tumor specimens may be associated with increased tumor-infiltrating lymphocytes, although due to the high level of expression of PD-L1, patients would still possess poorer prognosis.

ADAMTS13 is a metalloprotease that is crucial in the prevention of spontaneous microvascular thrombosis (23). ADAMTS13 has been found to play a role in the regulation of leukocyte adhesion and extravasation during 
inflammation $(23,24)$. Tumor-associated inflammation may induce the expression of various molecules in tumor sites and create a microenvironment that promotes cancer development (25). Since our data suggest that expression of ADAMTS13 confers further prognostic value to PD-L1 high bladder cancer, we find it possible that ADAMTS13-modulated inflammation may impair PD-L1-mediated cancer immunosurveillance. Future studies may be useful to determine the interrelationship of ADAMTS13 and PD-L1 in the progression of bladder cancer.

In conclusion, our data suggest that PD-L1 plays an important role in bladder cancer progression, and that PD-L1 is a promising therapeutic target for bladder cancer.

\section{Acknowledgements}

This study was supported by the National Science Foundation for Young Scientists of China (grant no. 31301172), the Natural Science Foundation of Fujian Province (grant no. 2014J01122), and the University of Macau Start-Up Research Grant \& Multi-Year Research Grant (grant no. SRG2014-00006-FHS \& MYRG2015-00065-FHS respectively). We would like to thank Omic Science and Technology Limited for supporting the gene analysis in the present study.

\section{References}

1. Liakou CI, Narayanan S, Ng Tang D, Logothetis CJ and Sharma P: Focus on TILs: Prognostic significance of tumor infiltrating lymphocytes in human bladder cancer. Cancer Immun 7: 10, 2007.

2. Redelman-Sidi G, Glickman MS and Bochner BH: The mechanism of action of BCG therapy for bladder cancer - a current perspective. Nat Rev Urol 11: 153-162, 2014.

3. Alexandroff AB, Jackson AM, O'Donnell MA and James K: BCG immunotherapy of bladder cancer: 20 years on. Lancet 353 : 1689-1694, 1999.

4. Chen DS, Irving BA and Hodi FS: Molecular pathways: Next-generation immunotherapy - inhibiting programmed death-ligand 1 and programmed death-1. Clin Cancer Res 18: 6580-6587, 2012.

5. Nakanishi J, Wada Y, Matsumoto K, Azuma M, Kikuchi K and Ueda S: Overexpression of B7-H1 (PD-L1) significantly associates with tumor grade and postoperative prognosis in human urothelial cancers. Cancer Immunol Immunother 56: 1173-1182, 2007.

6. Inman BA, Sebo TJ, Frigola X, Dong H, Bergstralh EJ, Frank I, Fradet Y, Lacombe L and Kwon ED: PD-L1 (B7-H1) expression by urothelial carcinoma of the bladder and BCG-induced granulomata: Associations with localized stage progression. Cancer 109: 1499-1505, 2007.

7. Boorjian SA, Sheinin Y, Crispen PL, Farmer SA, Lohse CM, Kuntz SM, Leibovich BC, Kwon ED and Frank I: T-cell coregulatory molecule expression in urothelial cell carcinoma: Clinicopathologic correlations and association with survival. Clin Cancer Res 14: 4800-4808, 2008.
8. Xylinas E, Robinson BD, Kluth LA, Volkmer BG, Hautmann R, Küfer R, Zerbib M, Kwon E, Thompson RH, Boorjian SA, et al: Association of T-cell co-regulatory protein expression with clinical outcomes following radical cystectomy for urothelial carcinoma of the bladder. Eur J Surg Oncol 40: 121-127, 2014.

9. Powles T, Vogelzang NJ, Fine GD, Eder JP, Braiteh FS, Loriot Y, Zambrano CC, Bellmunt J, Burris HA, Teng SM, et al: Inhibition of PD-L1 by MPDL3280A and clinical activity in pts with metastatic urothelial bladder cancer (UBC). J Clin Oncol (ASCO Meeting Abstracts) 32: 5011, 2014.

10. Lee JS, Leem SH, Lee SY, Kim SC, Park ES, Kim SB, Kim SK, Kim YJ, Kim WJ and Chu IS: Expression signature of E2F1 and its associated genes predict superficial to invasive progression of bladder tumors. J Clin Oncol 28: 2660-2667, 2010.

11. Sjödahl G, Lauss M, Lövgren K, Chebil G, Gudjonsson S, Veerla S, Patschan O, Aine M, Fernö M, Ringnér M, et al: A molecular taxonomy for urothelial carcinoma. Clin Cancer Res 18: 3377-3386, 2012.

12. Lindgren D, Sjödahl G, Lauss M, Staaf J, Chebil G, Lövgren K, Gudjonsson S, Liedberg $\mathrm{F}$, Patschan $\mathrm{O}$, Månsson W, et al: Integrated genomic and gene expression profiling identifies two major genomic circuits in urothelial carcinoma. PLoS One 7: e38863, 2012.

13. Kulbe H, Levinson NR, Balkwill F and Wilson JL: The chemokine network in cancer - much more than directing cell movement. Int J Dev Biol 48: 489-496, 2004.

14. Corthay A: Does the immune system naturally protect against cancer? Front Immunol 5: 197, 2014.

15. Kyi $\mathrm{C}$ and Postow MA: Checkpoint blocking antibodies in cancer immunotherapy. FEBS Lett 588: 368-376, 2014.

16. Langer CJ: Emerging immunotherapies in the treatment of non-small cell lung cancer (NSCLC): The role of immune checkpoint inhibitors. Am J Clin Oncol: Mar 28, 2014 (Epub ahead of print).

17. Eruslanov E, Neuberger M, Daurkin I, Perrin GQ, Algood C, Dahm P, Rosser C, Vieweg J, Gilbert SM and Kusmartsev S: Circulating and tumor-infiltrating myeloid cell subsets in patients with bladder cancer. Int J Cancer 130: 1109-1119, 2012.

18. Urquidi V, Kim J, Chang M, Dai Y, Rosser CJ and Goodison S: CCL18 in a multiplex urine-based assay for the detection of bladder cancer. PLoS One 7: e37797, 2012.

19. Goodison S, Chang M, Dai Y, Urquidi V and Rosser CJ: A multianalyte assay for the non-invasive detection of bladder cancer. PLoS One 7: e47469, 2012.

20. Miyake M, Ross S, Lawton A, Chang M, Dai Y, Mengual L, Alcaraz A, Giacoia EG, Goodison S and Rosser CJ: Investigation of CCL18 and A1AT as potential urinary biomarkers for bladder cancer detection. BMC Urol 13: 42, 2013.

21. Zhang L, Conejo-Garcia JR, Katsaros D, Gimotty PA, Massobrio M, Regnani G, Makrigiannakis A, Gray H, Schlienger K, Liebman MN, et al: Intratumoral T cells, recurrence, and survival in epithelial ovarian cancer. N Engl J Med 348: 203-213, 2003.

22. Chen DS and Mellman I: Oncology meets immunology: The cancer-immunity cycle. Immunity 39: 1-10, 2013.

23. Gandhi C, Khan MM, Lentz SR and Chauhan AK: ADAMTS13 reduces vascular inflammation and the development of early atherosclerosis in mice. Blood 119: 2385-2391, 2012.

24. Chauhan AK, Kisucka J, Brill A, Walsh MT, Scheiflinger F and Wagner DD: ADAMTS13: A new link between thrombosis and inflammation. J Exp Med 205: 2065-2074, 2008.

25. Atsumi T, Singh R, Sabharwal L, Bando H, Meng J, Arima Y, Yamada M, Harada M, Jiang JJ, Kamimura D, et al: Inflammation amplifier, a new paradigm in cancer biology. Cancer Res 74: 8-14, 2014. 